LCLS-TN-05-15

August 2005

\title{
Wakefield Calculations for Radiation Stopper 1 (RST1)*
}

\author{
Karl Bane, Cecile Limborg-Deprey, Cho-Kuen Ng \\ SLAC, Stanford, CA 94309, USA
}

${ }^{*}$ Work supported in part by the DOE Contract DE-AC02-76SF00515. This work was performed in support of the LCLS project at SLAC. 


\section{SUMMARY}

The main result of this note is that no wakefield mitigation is required for the Radiation Stopper (RST1) in the LCLS injector.

The RST1 geometry is not symmetric in the vertical direction, and we derive a slight modification to the diffraction model wake for a cylindrically symmetric (2D) cavity that can be used for this problem. Performing a full 3D MAFIA calculation for the nominal $1 \mathrm{~mm}$ (rms) long bunch, we show that the modified diffraction model well describes the wakefields generated in RST1. The results imply an on-axis emittance growth of $0.0075 \%$, well below the $0.5 \%$ tolerance threshold [1]. To reach the $0.5 \%$ threshold the beam would need to be mis-steered by a large amount $-7 \mathrm{~mm}$ - from the axis. One reason that the effect is small is that the beta functions at the RST1 are small.

\section{GEOMETRY OF RST1}

Two (collinear) beam pipes of radius $0.435 "$ (11 $\mathrm{mm})$, whose axis define the $z$ axis, impinge on the walls of a cylindrical metal tank, with axis oriented vertically (the $y$ direction). The tank has diameter 5.76" (146 mm) and height 5.76" (146 mm). Inside the tank (and aligned with its axis) is the radiation stopper itself, a solid stainless steel cylinder of 4" (102 mm) diameter. The stopper is nominally located above the beam pipe axis (in the $+y$ direction) with the bottom surface approximately even with the top surface of the beam pipe. The layout is shown in Fig. 1.

We are interested in the strength of the transverse wakefields excited by the bunch in RST1. We use MAFIA to perform 3D short-range wake calculations [2]. The MAFIA model closely follows the geometry of Fig. 1, with the stopper in its nominal position. In Appendix A we develop a modification of the diffraction model of cavity wakes [3] that allows us to obtain an analytical estimate that we compare with the MAFIA result. 


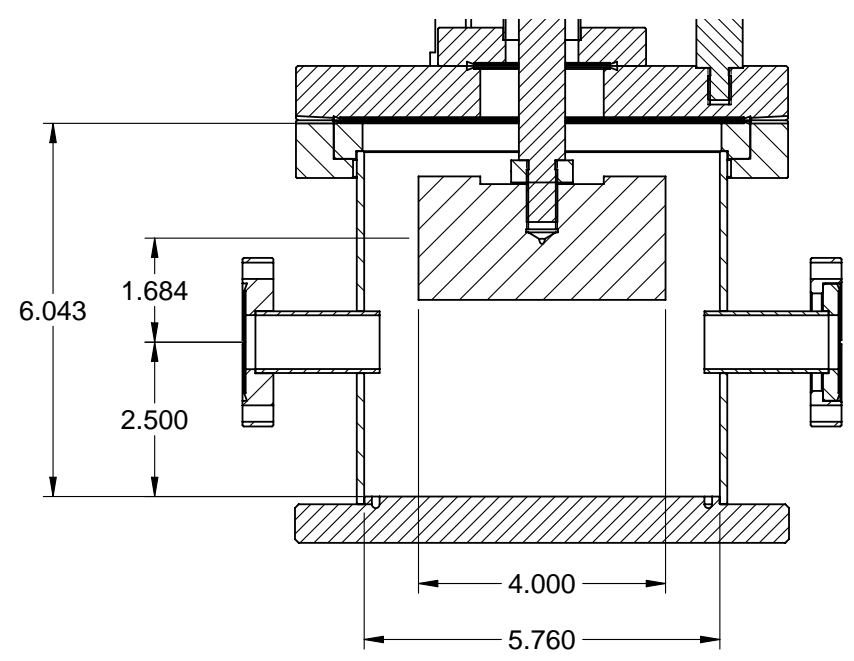

FIG. 1: Layout of radiation stopper RST1 in a vertical (y)/longitudinal $(z)$ projection on the horizontal $(x)$ symmetry plane. Dimensions are given in inches.

\section{CALCULATIONS}

Consider first a cylindrically symmetric cavity with beam pipes as a model of the RST1 tank alone, without stopper (see Fig. 2). [The tank itself is not cylindrically symmetric with respect to the beam pipe axis; nevertheless, since the tank is large, with its walls far from the beam axis, such a model still can be used.] When a short bunch $\sigma_{z} \ll a$ (with $\sigma_{z}$ the rms bunch length, $a$ the beam pipe radius) traverses the model cavity, wakefields due to diffraction radiation are generated at the transitions between the beam pipe and the cavity walls. If, in addition, the cavity is deep (the cavity radius $b \gtrsim a+\sqrt{2 g \sigma_{z}}$ with $g$ the length of cavity) then the so-called "diffraction model" of cavity wakes applies. The average of the transverse wake for a Gaussian bunch is given by [3]

$$
\left\langle W_{\perp}\right\rangle=(0.14) \frac{Z_{0} c}{a^{3}} \sqrt{g \sigma_{z}},
$$

with $Z_{0}=377 \Omega$ and $c$ the speed of light. The rms of the transverse wake $\left(W_{\perp}\right)_{r m s}=$ $0.77\left\langle W_{\perp}\right\rangle$.

We use MAFIA to compute the short-range wake for the actual (3D) geometry of the RST1 tank plus beam pipes [4], for (nominal) bunch length, $\sigma_{z}=0.95 \mathrm{~mm}$. We find that the shape of the wake (not shown) agrees well with that of the diffraction model, with an 


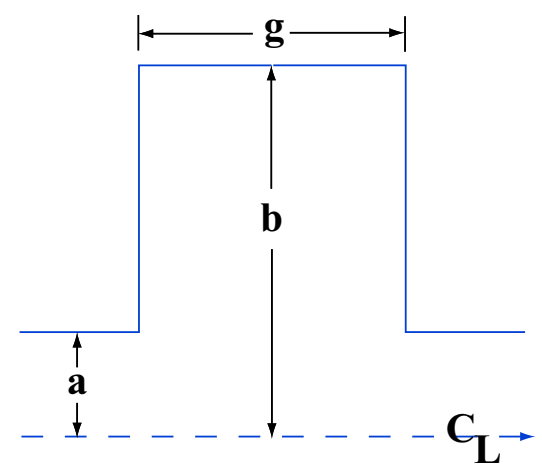

FIG. 2: The cylindrically symmetric cavity with beam pipes of the diffraction model.

average $\left\langle W_{x}\right\rangle=115 \mathrm{~V} / \mathrm{pC} / \mathrm{m}$. Note that the tank is large enough so that its outer wall dimensions play no role in the result. For the analytical model, taking $a=11 \mathrm{~mm}$ and $g=152.3 \mathrm{~mm}$, we obtain $\left\langle W_{x}\right\rangle=145 \mathrm{~V} / \mathrm{pC} / \mathrm{m}$, in reasonable agreement with the numerical result.

When including the stopper we are left in $y$ with an asymmetric cavity, which means that the wake is non-zero even on the beam pipe axis. Since the asymmetric cavity comprises a series of deep cavities, the shape of the wake remains the same (i.e. as the diffraction model wake); only the scale factor changes. In Appendix A we show how to approximately find the scale factor for the real, asymmetric RST1 geometry using the 2D diffraction model. The average of our result is

$$
\left\langle\tilde{W}_{y}\right\rangle=(0.14) \frac{Z_{0} c \sigma_{z}^{1 / 2}}{4 a^{2}}\left(-\sqrt{g}+2 \sqrt{g_{p}}\right)
$$

where the symbol $\tilde{W}_{y}$ is used to indicate an on-axis wake (with units $[\mathrm{V} / \mathrm{pC}]$ ). The wake contains kick contributions of two cavities on either side of the stopper, with gap $g_{p}=22 \mathrm{~mm}$ (in the $+y$ direction), and one cavity of gap $g=152.3 \mathrm{~mm}$ (in the $-y$ direction). We plot, in Fig. 3, the wake as given by MAFIA and compare with our modified diffraction model; we note good agreement. For MAFIA the average wake, $\left\langle W_{y}\right\rangle=-0.10 \mathrm{~V} / \mathrm{pC}$ (a downward kick), for our model $\left\langle W_{y}\right\rangle=-0.095 \mathrm{~V} / \mathrm{pC}$.

The relative emittance growth, if small, is given by $\Delta \epsilon / \epsilon \approx \frac{1}{2} \sigma_{\theta}^{2} \beta / \epsilon$, with $\sigma_{\theta}=W_{r m s} e N / E$. Taking charge $e N=1 \mathrm{nC}$, energy $E=135 \mathrm{MeV}$, emittance $\epsilon=3.7 \mathrm{~nm}$, and beta function $\beta=1.5 \mathrm{~m}$, we find that for average wake $\left\langle\tilde{W}_{y}\right\rangle=-0.10 \mathrm{~V} / \mathrm{pC}$ [which implies $\left(W_{y}\right)_{r m s}=$ 




FIG. 3: MAFIA calculation of $\tilde{W}_{y}$ for beam stopper RST1 (blue) and the analytical model result, Eq. A4 (red).

$0.077 \mathrm{~V} / \mathrm{pC}], \Delta \epsilon / \epsilon=0.007 \%$, which is much lower than the $0.5 \%$ limit of our budget. In the $x$ direction the cavity is symmetric, and the symmetric diffraction model can be applied. The average of the wake is $\left\langle W_{x}\right\rangle=140 \mathrm{~V} / \mathrm{pC} / \mathrm{m}$. To reach our $0.5 \%$ emittance growth limit the beam would need to be mis-steered by $7 \mathrm{~mm}$ from the axis, and about the same is true in $y$. Therefore, wakefields in the RST1 stopper are not a problem for the LCLS.

\section{APPENDIX A: DIFFRACTION MODEL APPLIED TO THE RST1 STOPPER}

We here demonstrate that the RST1 wakes are diffraction wakes, and that they can be approximated using slightly modified versions of known formulae for cylindrically symmetric structures. We are primarily interested in the strength of the transverse wakefields excited in RST1. However, to conclusively demonstrate that the diffraction model of wakefields applies we begin by investigating the longitudinal wakes. All the formulae for the symmetric case can be found in Ref. [3].

\section{Longitudinal Wake}

Consider a cylindrically symmetric cavity with beam pipes. When a short bunch $\sigma_{z} \ll a$ (with $\sigma_{z}$ the rms bunch length, $a$ the beam pipe radius) traverses the cavity, wakefields due to diffraction radiation are generated at the transitions between the beam pipe and the cavity walls. If, in addition, the cavity radius is sufficiently large $\left(b \gtrsim a+\sqrt{2 g \sigma_{z}} ; b\right.$ radius 
and $g$ length of cavity), then the diffraction model of cavity wakes applies. For a Gaussian bunch the longitudinal wakefield is given by [3]

$$
W_{z}\left(s, \sigma_{z}\right)=-\frac{Z_{0} c}{\sqrt{2} \pi^{2} a} \sqrt{\frac{g}{\sigma_{z}}} f\left(s / \sigma_{z}\right),
$$

with

$$
f(x)=\frac{1}{\sqrt{2 \pi}} \int_{0}^{\infty} \frac{e^{(y-x)^{2} / 2}}{\sqrt{y}} d y .
$$

A positive bunch wake implies energy gain; $s$ is position within the bunch with $s<0$ at the head of the bunch; with $Z_{0}=377 \Omega$ and $c$ the speed of light. The average of the function $f(x)$ (when weighted by a Gaussian) is $\langle f\rangle=0.72$ and the rms is $f_{r m s}=0.29$. Using MAFIA to compute the short-range wakes for the RST1 tank alone (without absorber), taking $a=11 \mathrm{~mm}, g=152.3 \mathrm{~mm}$, and $\sigma_{z}=0.95 \mathrm{~mm}$, we find that the shape of the wake agrees well with the diffraction model. As for the average, MAFIA obtains $\left\langle W_{z}\right\rangle=7.0 \mathrm{~V} / \mathrm{pC}$, the diffraction model gives $6.8 \mathrm{~V} / \mathrm{pC}$.

When including the absorber we are left with an asymmetric cavity. We can, nevertheless, use the symmetric-cavity diffraction results to estimate the wakefields for the real, asymmetric geometry. Important for this to work, is the fact that the beam pipe is round (with $\sigma_{z} / a \ll 1$ ) and that the cavity is itself composed of several deep sub-cavities. The radiation is at high frequency and generated locally at the beam pipe/wall transitions, and what happens in the upward $[+y]$ direction in the cavity is relatively independent of what happens in the downward $[-y]$ direction. The asymmetric cavity will have a longitudinal (s) dependence of the wakes (both longitudinal and transverse) that is the same as for the symmetric case; only the scale factor changes.

For the RST1 with absorber the longitudinal wake can be approximated as the average of: what was obtained before without the absorber (the lower-half contribution) and twice the effect for a cavity with gap $g_{p}=22 \mathrm{~mm}$ (the upper-half contribution). The wake becomes

$$
W_{z}=-\frac{Z_{0} c}{\sqrt{2} \pi^{2} a} \sqrt{\frac{g}{\sigma_{z}}} f\left(s / \sigma_{z}\right)\left(\frac{1}{2} \sqrt{g}+\sqrt{g_{p}}\right) .
$$

In Fig. 4 we plot the longitudinal wake for RST1 as obtained by MAFIA, and compare with the analytical result; we see that the agreement is good. The weighted average $\left\langle W_{z}\right\rangle=$ $-5.9 \mathrm{~V} / \mathrm{pC}$ for the MAFIA result and $-5.9 \mathrm{~V} / \mathrm{pC}$ for the analytical formula. 




FIG. 4: MAFIA calculation of $W_{z}$ for beam stopper RST1 (blue) and the analytical model result, Eq. A1 (red).

\section{Transverse Wake}

For a Gaussian bunch in a cylindrically symmetric structure the transverse wake, according to the diffraction model, is given by [3]

$$
W_{\perp}\left(s, \sigma_{z}\right)=\frac{2^{3 / 2} Z_{0} c}{\pi^{2} a^{3}} \sqrt{g \sigma_{z}} h\left(s / \sigma_{z}\right),
$$

with

$$
h(x)=\frac{1}{2} \int_{0}^{x} d y f(y) .
$$

For the function $h(x)$ the weighted average $\langle h\rangle=0.49$ and the rms $h_{r m s}=0.37$. For the stopper tank alone the MAFIA result is $\left\langle W_{\perp}\right\rangle=115 \mathrm{~V} / \mathrm{pC} / \mathrm{m}$, that of the diffraction model is $145 \mathrm{~V} / \mathrm{pC} / \mathrm{m}$.

For the real RST1 structure in the vertical (y) direction, since the geometry is asymmetrical, there is a wake even on the beam pipe axis. For a structure with a cavity asymmetrically placed (on one side of the beam axis only), the wake's transverse dependence must be $a^{-2}$, with $a$ the distance from the beam axis to the edge of the cavity. To find the coefficient, note that the transverse dependence - for the symmetric case - of $W_{y} y$ is $y / a^{3}$, which can be expanded in Taylor series as

$$
\frac{y}{a^{3}} \approx \frac{1}{4}\left[\frac{1}{(a-y)^{2}}-\frac{1}{(a+y)^{2}}\right] .
$$

This suggests that, for e.g. a cavity in only the $+y$ direction, $y / a^{3}$ in $W_{y} y$ using Eq. A4 should be replaced by $1 /\left(4 a^{2}\right)$. Thus, as estimate to the average of the RST1 transverse 
wake we take

$$
\tilde{W}_{y}=\frac{2^{3 / 2} Z_{0} c \sigma_{z}^{1 / 2}}{4 \pi^{2} a^{2}} h\left(s / \sigma_{z}\right)\left(-\sqrt{g}+2 \sqrt{g_{p}}\right)
$$

(the symbol $\tilde{W}_{y}$ is used to indicate an on-axis wake, with units $[\mathrm{V} / \mathrm{pC}]$ ). We plot the wake as given by MAFIA and the analytical model in Fig. 3 and note good agreement. The average value for the MAFIA result is $-0.10 \mathrm{~V} / \mathrm{pC}$ (a downward kick) and the analytical model gives $-0.095 \mathrm{~V} / \mathrm{pC}$. The excellent agreement may be somewhat accidental: remember that for the symmetric cavity the agreement between MAFIA and the analytical model (in the transverse direction) was only to $25 \%$. Finally, from an additional MAFIA calculation we find that by moving the beam $1 \mathrm{~mm}$ upward, the transverse wake can approximately be cancelled, which is also consistent with the diffraction model.

\section{REFERENCES}

[1] C. Limborg-Deprey, K. Bane, "Impedance Budget for the Injector," LCLS-TN-05-17, 2005.

[2] The MAFIA Collaboration, User guide, CST-GmbH, Darmstadt, Germany.

[3] K.L.F. Bane and M. Sands, in Part. Accel. 25:73 (1990).

[4] Note that for diameter of the tank, $152.3 \mathrm{~mm}$ - a number $4 \%$ larger than in the designwas inadvertently used in the simulations. 